\title{
A Method of Gene Expression Data Partitioning for the Construction of Context-Sensitive PBNs Le $\mathrm{Yu}^{*}$ and Stephen Marshall
}

\author{
Address: Department of Electronic and Electrical Engineering, University of Strathclyde, Glasgow, UK. \\ Email: Le Yu* - l.yu@eee.strath.ac.uk \\ * Corresponding author
}

from BioSysBio: Bioinformatics and Systems Biology Conference

Edinburgh, UK, 14-15 July 2005

Published: 2I September 2005

BMC Bioinformatics 2005, 6(Suppl 3):P29

This paper presents a new method of fitting probabilistic Boolean networks (PBN) to gene expression data sequences.

In recent years biological microarray technology has emerged as a high-throughput data acquisition technology in bioinformatics. In conjunction with this, a significant amount of attention has been focused on modelling genetic regulatory networks from gene expression data. However, with the appearance of various sources of data, the previous deterministic models are not able to capture the time-varying dependencies between the different components in the gene regulatory network.

Recently, a new modelling approach defined as ContextSensitive Probabilistic Boolean Networks (Context-Sensitive PBNs) was proposed for inferring genetic regulatory networks from gene expression data with different contexts. The Context-Sensitive PBNs model is a collection of Boolean Networks with fixed predictors in a time-course. Each constituent Boolean Network governs gene activity in a time horizon until a switch point occurs corresponding to some random external event.

For the construction of Context-Sensitive PBNs, it is essential to be able to partition gene expression data into sections corresponding to different contexts of the underlying model. We propose a method for partitioning the sample gene expression data with multi-context into different segments according to the context in a certain time horizon. This is carried out by identifying the switch points between functional states of the data sequence, and separating the given data sequence into segments corresponding to different contexts. Our approach is based on the analysis of the gene activity profile (GAP) formed by the states of individual genes at each time step.

Results from the method will be demonstrated which will identify the points in the sequence where the parameters of the Boolean model switch, the precise functions which regulate the network and the genes on which they depend. 